\title{
LESSON 10
}

MARGINS:

Left, Pica 20; Elite 30;

Right, moved out of the way.

PAPER: Against the paper guide at 0 on the scale on the left. Turn up 7 single lines.

Check the 'ready-to-type' position: feet, body, fingers and eyes.

\section{Warm up}

Bounce your right thumb off the space bar.

Return the carriage smartly.

Keep your eyes on the copy.

Tap the keys sharply.

\section{Left shift key}

Keep the ds fingers in position as well if possible.

Keep your eyes on the copy.

Bounce your right thumb off the space bar.

\section{Full stop (.) key}

Practise the reach until you can type the full stop with the I finger without looking down.

Keep the $\mathrm{j}$ or ; finger in position.

\section{q key}

Practise the reach until you can type $q$ with the a finger without looking down.

Keep the fds fingers in position as you type q. Try not to lift your elbow.

Keep your eyes on the copy.

\section{Consolidation}

Check the 'ready-to-type' position. Return the carriage smartly. Keep your eyes on the copy. Try to type each line in $1 / 2$ minute. Use the paper release lever when taking the paper out of the machine.

Centre and lock the carriage.

Cover the machine.

UNIT 3

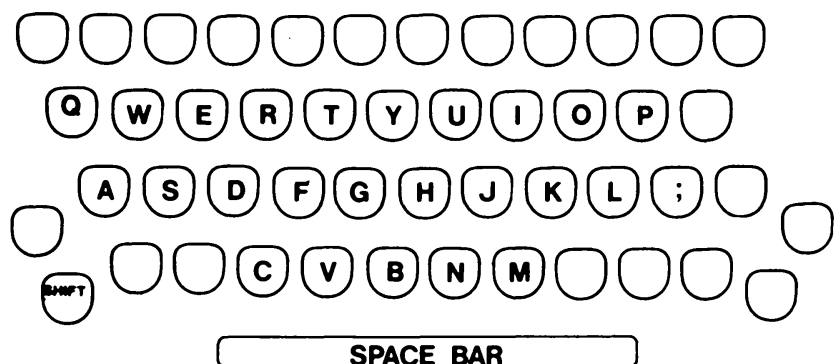

Remember to leave a blank line between sections.

Type the lines indicated by your teacher 3 times each.

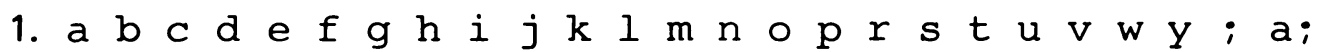

2. a very kind old man put the safe by the west wall;

3. that is not so very far from the two up at the top

4. kindly pass the jars of pickled onions now please;

5. by the side of the lake they just saw him dip both

Type these lines as many times as your teacher asks. Keeping the $f$ finger in position, reach with the little finger of the.left hand to the shift key and hold it down to its fullest extent while the right hand types the capital letter. Return the little finger to the a key.

6. Jan Jan Kate Kate Ian Ian Paul Paul Karl Karl Ken;

7. Jan and Pat and Ida and Ken and Jim and June went;

8. His name is John Mark Nevil Henry Oliver Patterson

Gently type the full stop. Type this line as many times as your teacher asks.

9. $1111.1 \ldots . .1 .1 .1 j 1 . j$ jl. jl. .1.1.1.1 l.j. l.l

Type this line 3 times. Leave two spaces after a full stop at the end of a sentence.

10. I am. You are. He is. Just once then. Join it.

Type this line as many times as your teacher asks.

11. aaa aqa aqa qqq aqu aqu aqa aqfq aqu aqu aqfa aqfa Type the lines indicated by your teacher 3 times each.

12. qu qui quite qu quiet equ equal qui quip squ squaw 13. quip quip quota quota sequel sequel squint squints

14. Just squash it quickly; here is the queen of Iraq.

15. Mike squashed a quire quota of quaint quick limes.

Type each line 3 times. Try to build up speed each time you repeat a line.

16. John has just enquired about the kipper knife set. 17. Now we can have our trek. Peter can come as well.

18. Henry has a squint. Pat uses a new glass tankard. 19. You should just see the cave Keith and Paul found. 20. Last night was quite a party after the hard tests. 